\title{
174. Scattering Simulation of Laser Beam Propagating through Fog
}

\author{
Tatsuo SHIINA, Takashi MITSUGI, Sohachi MORISHIMA and Koichi IKEDA \\ ( $\mathrm{T}$ ok yo R i k a D a i g a k u )
}

\section{INTRODUCTION}

To know exact slant visual range, the measurement of backscattered light of laser beam using a laser radar system is one of the important practical method. At the Hamburgairport, one of these systems was installed and has been used to measure the slant visual range. Several papers concerning this system have been reported, however, these represent only measured data, and are not analyzed scattering characteristics.

In our laboratory, a lot of measured data have been stored by using our laser radar system for more than 15 years under various weather conditions. The traditional laser radar equation, which has been constructed under the assumption that transmitted beam would propagate as a parallel beam and would not diverge irrespective of weather conditions, has been used to analyze these data at early stage of our study. It was, however, found that the received intensity of the backscattered light does not frequently follow the current equation because of the divergence of the transmitted beam.

So, considering the spread of the transmitted beam, the equation has been developed to simulate to the measured data. As a result of the analysis, it is found that the estimated visibility corresponds to the real one, and the scattering of the transmitted beam becomes large in the order of rain, fog, drizzle to snow.

To confirm the scattering of the transmitted beam experimentally, the scattering characteristics of the transmitted beam are measured and ascertained, and the spread functions are derived from these data. Then the simulation of the transmitted laser beam spread by scattering is studied with the aid of a computer.

\section{EXPERIMENT OF SCATTERING CHARACTERISTICS OF TRANSMITTED LASER BEAM}

To examine the scattering characteristics of the transmitted beam, Fourier optical system is constructed in a fog chamber. Stratified fog $(6 \mathrm{~cm})$ is generated with an ultrasonic fog generator at the center of this system. The laser beam passes through this stratified fog. The angle characteristics of the scattered beam are detected at the focal length of the lens. The angle component of the scattering beam is transiormed to the position on the screen. The model of the angle characteristics is shown in Fig. 1.

The point spread functions of the spread angle are derived from the scattered cross section of the transmitted beam, and these functions are superimposed on each point of the cross section of the transmitted beam without scattering. The example of the point spread function is shown in Fig. 2. This superimposed cross section of the transmitted beam corresponds well to the scattered cross section obtained by the experiment.

\section{SCATTERING SIMULATION OF TRANSMITTED BEAM THROIJGH FOG}

The spread functions are applied to the scattering simulation of the transmitted beam through fog. The empirical formula is derived from the correlation between the transmittance through fog and the spread function. This scattering simulation is formed by superimposing the point spread functions on each point of the cross section of the transinitted beam without fog as the equations (1). 


$$
\begin{aligned}
& \mathbf{I}_{1}(\theta)=\int \mathbf{F}\left(\theta-\theta^{\prime}\right) \cdot \mathbf{I}_{0}\left(\theta^{\prime}\right) \mathbf{d} \theta^{\prime} \\
& \mathbf{I}_{2}(\theta)=\int \mathbf{F}\left(\theta-\theta^{\prime}\right) \cdot \mathbf{I}_{1}\left(\theta^{\prime}\right) \mathbf{d} \theta^{\prime} \\
& \vdots \\
& \mathbf{I}_{N}(\theta) \stackrel{=}{=} \mathbf{F}\left(\theta-\theta^{\prime}\right) \cdot \mathbf{I}_{\mathrm{N}-1}\left(\theta^{\prime}\right) \mathbf{d} \theta^{\prime}
\end{aligned}
$$

Where $\mathbf{I}_{0}(\theta)$ is an incident beam, $\mathbf{I}_{\mathrm{N}}(\theta)$ is the latter pattern of the scattered beam, $\mathbf{I}_{\mathrm{N}-1}(\theta)$ is the former pattern of that, and $\mathbf{F}(\theta)$ is the point spread function. $\theta$ is a relative angle of the incident beam, and $\theta^{\prime}$ is that of the outlet beam. The index $\mathbf{N}$ is a step number of stratified fog This concept is shown in Fig. 3 and the flowchart of this simulation program is shown in Fig. 4. The measured pattern (Stratified Fog : $24 \mathrm{~cm}$ ) corresponds to the superimposed pattern, which is convolutionally integrated the incident pattern without fog four times by the spread function estimated by the empirical formula. The spatial spread of the transmitted beam along the pass can be calculated by piling up the spread functions. The calculated pattern (Stratified fog : $1 \mathrm{~m}$ ) is also shown in Fig. 5. It is possible to apply this simulation to the laser radar system by extending the fog space for a long distance. In the real atmosphere, it is possible that the transmitted laser beam diverges to the extent over the receiving area of the receiving telescope. Therefore, the traditional laser radar equation does not correspond to the intensity received by laser radar system in low visibility conditions. On the other hand, the new equation considered the divergence of the transmitted beam will represent the received intensity variation.

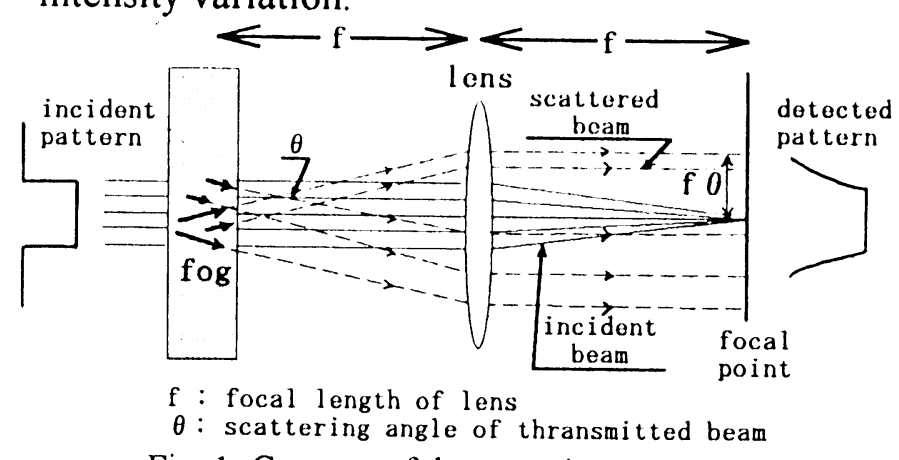

Fig. 1. Concept of the experiment.

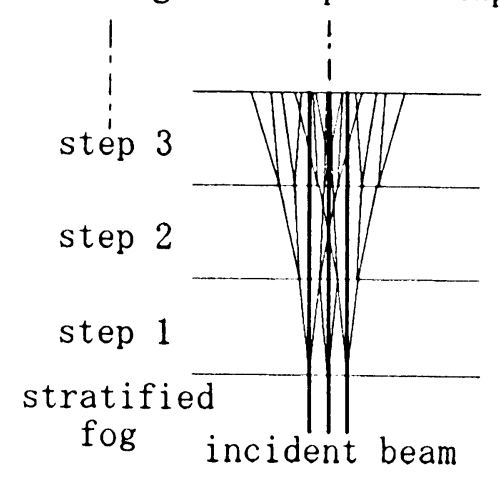

Fig. 3. Scattering model of the transmitted beam.

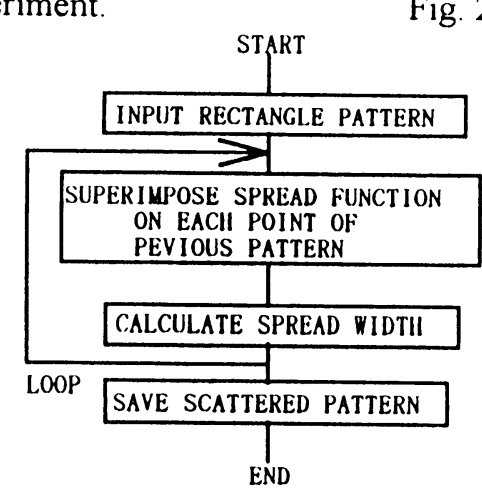

Fig. 4. Flowchart of scattering simulation.

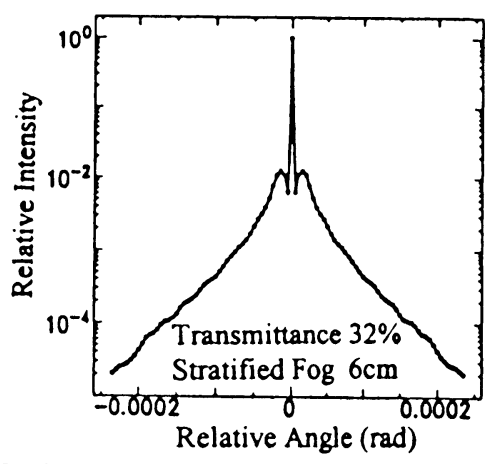

Fig. 2. Example of the point spread function.

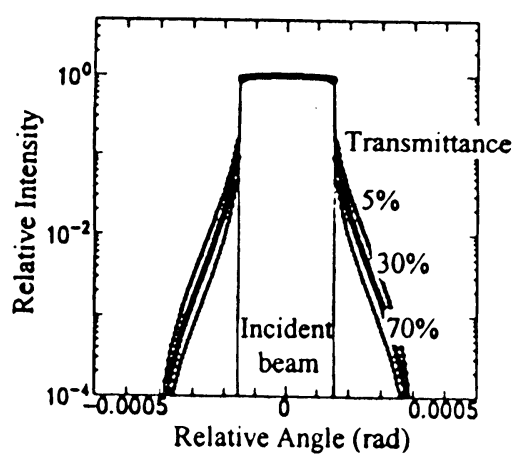

Fig. 5. Result of simulation at $1 \mathrm{~m}$.

\section{CONCLUSIONS}

It is possible to simulate the divergence of the transmitted laser beam by using the point spread functions, which is derived by the experimental data. Then it is found that the transmitted beam is scattered by fog and gradually spreads. To apply this simulation to the laser radar system by extending the fog space for a long distance, it would be considered the spatial width of the beam cross section in addition to the scattering angle. 